\section{A right hemisphere basis for calculation in the human brain*}

\author{
STUART J. DIMOND and J. GRAHAM BEAUMONT \\ University College, Cardiff, Wales
}

Ss were asked to perform calculations with two-digit numbers presented by a divided visual-field technique to either the right or left hemisphere of the brain and to match the results with further numbers, responding bimanually. There was no interhemispheric difference in response latency, but analysis of errors showed that more errors were associated with presentation to the left hemisphere for subtraction problems and for all problems taken together. This apparent right-hemisphere superiority is contrasted with traditional views.

Observations of the lesions leading to dysfunction in numerate abilities have, classically, led to the left parietal lobe being described as the site of such operations (Brain, 1964). Critchley (1953) reports that $86 \%$ of right-handed patients with unilateral cerebral lesions exhibiting acalculia have lesions of the left hemisphere, although Gloning, Gloning, \& Hoff (1968) argue that disturbances of this skill are more clearly related to the strategies employed in performance rather than the skill itself.

Sperry (1968) has suggested on the basis of the early split-brain cases that the ability to calculate appeared to be almost totally absent from the right hemisphere. It appeared, for example, that in nonverbal tests of calculation, the minor hemisphere was unable to perform a simple subtraction task employing two single digits. However, in some of the early patients the possibility of damage to the right hemisphere prior to surgery cannot be excluded, and it may be that to this can be attributed the deficit in calculation. In more recent work, Sperry \& Biersner ${ }^{1}$, using different testing procedures, have shown that moderately good addition, subtraction, and multiplication for at least simple numbers have been obtained, and Levy-Agresti (1968) also reports good evidence for the ability to calculate in the right hemisphere. The Ss of the last study were required to indicate the answer, using their left hand, to sums involving the product of numerals projected in the left visual field. The output factor appears to be an important one in limiting the expression of what the right hemisphere can perform.

What may be true of the split-brain patient, or the patient with a circumscribed lesion, may not be true

*The work reported here was carried out under tenure of Grant G969/96/C of the Medical Research Council. The authors are also indebted to Mr. P. Kinsey for his help in both the organization of apparatus and running of the experiment. of the individual with a normal intact brain. The study reported here set out to examine calculation functions in the normal brain by projecting numbers for addition and subtraction to either the right or left hemisphere using a divided visual-field technique. This study provides evidence of a functional superiority, not of the left hemisphere, as inferred from cases with brain lesions, but of the right or so-called minor hemisphere.

\section{METHOD}

Each of the 12 Ss, who were first-year undergraduates, was presented with the stimulus material by means of a divided visual field apparatus. The stimuli were back-projected onto one of four screens $(28 \times 44 \mathrm{~cm}, 63 \mathrm{~cm}$ from the S) which were arranged together, with partitions between these screens and the S's eyes so that, as the S gazed at a central fixation light, the material on Screen 1 (numbering from left to right) was directed at the nasal hemiretina of the left eye, that on Screen 2 at the nasal hemiretina of the right eye, that on Screen 3 at the temporal hemiretina of the left eye, and that on Screen 4 at the nasal hemiretina of the right eye. Thus, material appearing on Screens 1 and 2 (left visual field, LVF) was directed to the right hemisphere and that on Screens 3 and 4 (right visual field, RVF) to the left hemisphere (Dimond \& Beaumont, 1971). Stimuli were pairs of digits randomly selected and arranged in sets of three. On the first 40 trials, the $S$ was asked to press two microswitches once bimanually if the third digit pair equalled the sum of the first two digit pairs and twice if it did not equal this sum. For the second 40 trials, the task was to determine whether the third digit pair was equal to the difference between the first two, the response to be the same. Sets of digit pairs were organized so that on "addition" trials no sum was greater than 100 and on "subtraction" trials no difference was less than zero, half of the trials being "correct" and half "incorrect." Each sequence of three digit pairs was projected to either the right or left visual field (one digit per screen), randomly allocated to trials. The exposure time for each digit pair was $640 \mathrm{msec}$, and the interstimulus interval was $2,000 \mathrm{msec}$. Response latency was measured from the onset of the third digit pair to the earliest of the bimanual responses. Fixation on the central light was monitored and proved no problem for the Ss. The percentage of correct responses for each condition, as well as mean response latencies for correct and total responses, were calculated for each $S$ and then combined to yield group parameters.

There were no significant differences in response latency, as shown in Table 1 , between RVF and LVF trials on either addition or subtraction for correct or total responses, although correct responses were associated with a shorter response latency, as were responses to trials requiring the response of "correct." The percentage of correct responses as analyzed by field of presentation are given in Table 2 . It will be seen that while there is no statistically significant difference between the means for addition, right he misphere superiority is demonstrated for subtraction $(\mathrm{p}<.05$, $\mathrm{t}=2.244)$ and for total trials $(\mathrm{p}<.05$, $t=2.701)$. A significant difference was also observed, indicating the superior accuracy of responses to trials requiring the response "not correct." DISCUSSION

The argument could be expressed that information at the right hemisphere is transferred to the left for calculation to occur. This could be shown, for example, by increased response latencies, but, although the latencies at the right are slightly longer, they are not significantly so, nor do they approach significance. Moreover, the central fact which the results illustrate is that when task information is projected to the right hemisphere, fewer errors are made than when information is projected to the left. It is this which leads us to

Table 1

Mean Response Latencies for Correct and Total Responses (M sec) $\dagger$

\begin{tabular}{lll}
\hline & $\begin{array}{c}\text { Correct } \\
\text { Responses }\end{array}$ & $\begin{array}{c}\text { Total } \\
\text { Responses }\end{array}$ \\
\hline LVF & $584(300)$ & $613(333)$ \\
RVF & $620(307)$ & $655(338)$ \\
Yes* & $481(215)$ & $567(325)$ \\
No* & $698(354)$ & $702(347)$ \\
\hline
\end{tabular}

+Standard deviations giten in parentheses * Correct solution 
suggest a right-hemisphere basis for calculation.

That the difference between the hemispheres is only to be found on subtraction and on total trials is no doubt a result of the relative difficulty of the two tasks. The addition task being performed with relative ease fails to show the effect that occurs on a more difficult task. Whether the failure to find a difference in response latency between the hemispheres is a true indication of the structure of these functions or an artifact of the relatively long interstimulus interval which allowed much of the computation to be completed before the onset of the third digit pair cannot at present be determined.

The question arises as to why the idea that deficits in the function of calculation occur only in relation to left-hemisphere damage has had such a wide currency, and yet in the results reported here from a study of the normal intact brain we find the right to be superior certainly in the substance of calculation. One suggestion is that, in the past, calculation functions have often been tested by the capacity of the patient to verbalize the answer. If the machinery for calculation is preeminent in the right, a left-hemisphere lesion could act over a
Table 2

Percentage of Correct Responses Analyzed by Field of Presentation*

\begin{tabular}{lcc}
\hline & LVF & RVF \\
\hline Addition & 90.00 & 82.50 \\
& $(8.79)$ & $(12.52)$ \\
Subtraction & $\mathbf{8 4 . 5 8}$ & $\mathbf{7 8 . 7 5}$ \\
& $(9.16)$ & $(\mathbf{1 0 . 4 7 )}$ \\
Total & $\mathbf{8 7 . 2 9}$ & $\mathbf{8 0 . 6 3}$ \\
& $(\mathbf{8 . 4 9 )}$ & $(\mathbf{7 . 4 7 )}$ \\
\hline
\end{tabular}

*Standard deviations given in parentheses

wide area to dissociate the mechanism of calculation from the speech output system, thus preventing the patient from speaking the correct answer. Right-hemisphere lesions may not have this effect, for, although they interfere with a calculating register, there may be other registers to compensate. Left-hemisphere lesions interfere essentially with output, and thus some explanation of the effects can be provided.

The present results must cast doubt on traditional views of the calculation process which emphasized the essential nature of verbal involvement in the process or suggested a functional relationship with motor organization of the preferred hand (Critchley, 1953). It is clear that even if such factors are important in terms of the output system normally associated with calculation or with the early developmental acquisition of numerate abilities, they are not, in themselves, essential characteristics of the process of calculation. It is only possible to conclude that when a numerical task of relative difficulty is presented initially to either one of the two cerebral hemispheres, the process is completed more accurately when that hemisphere is the right rather than the left.

\section{REFERENCES}

BRAIN, LORD. Clinical neurology. 2nd ed. London: Oxford University Press, 1964. P. 101 .

CRITCHLEY, M. The parietal lobes. London: Edward Amold, 1953.

DIMOND, S. J., \& BEAUMONT, J. G. The use of two cerebral hemispheres to increase brain capacity. Nature, 1971, 232, 270.

GLONING, T., GLONING, K., \& HOFF, H. Neuropsychological symptoms and occipital lesions. Paris: Gauthier-Villars, 1968.

LEVY-AGRESTI, J. Ipsilateral projection systems and minor hemisphere function in man after neocommissurotomy. Anatomical Record, 1968, 160, 384.

SPERRY, R. W. Mental unity following surgical disconnection of the cerebral hemispheres. The Harvey Lectures, Series 62. New York: Academic Press, 1968 .

NOTE

1. As quoted in Sperry (1968). 